\title{
Calcium alternans is a global order-disorder phase transition. Robustness on Ryanodine Receptor release dynamics
}

\author{
Enrique Alvarez-Lacalle ${ }^{1}$, Angelina Peñaranda ${ }^{1}$, Inmaculada R. Cantalapiedra ${ }^{1}$, Yohannes \\ Shiferaw $^{2}$, Blas Echebarria ${ }^{1}$ \\ ${ }^{1}$ Universitat Politècnica de Catalunya, UPC, Spain \\ ${ }^{2}$ California State University-Northridge (CSUN), Los Angeles, United States
}

\begin{abstract}
Electromechanical alternans is a beat-to-beat alternation in the strength of contraction of a cardiac cell which appears often due to an instability of calcium cycling. The global calcium signal in cardiomyocytes is the result of the combined effect of several thousand micron scale domains called Calcium Release Units (CaRU), coupled through diffusion, where the flow of calcium among different cell compartments is regulated by stochastic signaling involving the ryanodine receptor $(R y R)$. Recently, numerical simulations have suggested that the transition from regular $\mathrm{Ca}$ cycling to alternans is an order-disorder phase transition consistent with the Ising universality class. Inside the cell, groups of CaRU form transient areas within the cell where alternans appear. However, global alternans appears only as a result of the synchronization of the oscillation phase among different subunits. We show here that this transition is indeed robust and universal upon changes in the behavior of the RyR. Using three different set of parameters for the transition rates among open, closed and inactivated states in the RyR, we show that different RyR behavior leads to the same type of order-disorder transition.
\end{abstract}

\section{Introduction}

Cardiac patients diagnosed with T-wave alternans, a beat-to-beat alternation in the shape of the electrocardiogram (ECG), are known to be at a higher risk of suffering from life threatening arrhythmias [1-3]. This beat-to-beat alternation in the strength of contraction of a cardiac cell is related either to a dysregulation of membrane voltage or, most commonly, to an instability of calcium (Ca) cycling dynamics in cardiac myocytes. When a cardiac cell is rapidly paced by a periodic stimulus it is known to exhibit complex aperiodic dynamics of both voltage and calcium. The most commonly observed behavior is the phenomenon of alternans where the action potential duration (APD) and $\mathrm{Ca}$ transient of a cardiac cell alternates from one beat to the next $[2,4,5]$. Earlier work showed that cellular alternans could arise from a period-doubling bifurcation associated to the nonlinear kinetics of ion channels regulating the membrane potential. However, later experimental studies revealed that alternans may also appear due to an instability of Ca cycling independently of the dynamics of membrane voltage $[3,6]$, thus stressing the important role of subcellular Ca signaling.

Calcium cycling is an intracellular process regulated by several thousand micron scale domains, referred to as $\mathrm{Ca}$ release units (CaRUs), where the flow of calcium in and out of the cell is regulated by stochastic signaling [7]. While an average (or mean field) description of intracellular calcium alternans leads naturally to a period doubling bifurcation when the pacing period is decreased, the underlying stochasticity of its multiple subcellular domains suggests a more complex behavior close to the transition point. An approach based on an average description focuses on the effects and causes of the nonlinear release, either by strong dependency of calcium release on SR load, or RyR recovery from inactivation [8]. On the contrary, an analysis of the local stochastic $\mathrm{Ca}$ dynamics focuses on the combination of stochasticity of $\mathrm{Ca}$ signaling and nearest neighbor interactions as sources of calcium alternans [7,9]. In this line, theoretical studies of $\mathrm{Ca}$ dynamics have attributed alternans to the combination of the underlying stochasticity of $\mathrm{Ca}$ signaling and the regenerative recruitment of $\mathrm{Ca}$ release due to nearest neighbor interactions [10], suggesting that $\mathrm{Ca}$ alternans is a direct consequence of the collective behavior of the thousands of signaling complexes in the cell.

Recently, a detailed model of stochastic $\mathrm{Ca}$ cycling showed that the transition from regular $\mathrm{Ca}$ cycling to alternans occurs via an order-disorder phase transition consistent with the Ising universality class [11]. This transition follows from the described features of the Ca cycling architecture, specially the coupling between signaling units due to $\mathrm{Ca}$ diffusion. Calcium signalling exhibits features that can be described using equilibrium statistical mechan- 
ics, where stochasticity at the ion channel level serves the role of temperature, while cellular diffusion leads to spatial cooperativity.

A consequence of this understanding of calcium alternans is that the appearance and nature of alternans must be similar even under very different local calcium dynamics as long as the local stochastic dynamics presents a strong non-linearity in the probability of calcium release upon some local variable of the CaRU in the release unit. Thus, very different RyR behavior can lead to the same transition as long as this nonlinearity is present. In this paper, we report that three different scenarios for the functioning of the RyR at the local level lead, all of them, to the same kind of order-disorder transition presented in previous works.

\section{Materials and methods}

We model a Z-plane of the cell as a two dimensional (2D) network of diffusively coupled compartments using the same equations and parameters indicated in the supplement material of [11] except for the transition rates of the RyR and a $20 \%$ reduction of the exchanger strength to obtain alternans at pacing of $4 \mathrm{~Hz}$. This model captures the local signal transduction via a direct simulation of 5 LCC channels in close proximity with 70 RyR channels in each CaRU. Each channel is modeled by simulating the stochastic channel transitions between states of experimentally derived Markovian schemes [See Fig. 1 ]. These junctions are coupled via diffusive currents in the cytosol and SR and buffers are added in the cytosol but not in the SR. To study the dynamics of Ca cycling we pace a $\mathrm{L} \times$ $\mathrm{L}$ array of signaling units using an action potential (AP) clamp. The numerical scheme makes use of a propagator and slaving procedure which prevents the numerical spurious generation or elimination of calcium due to numerical artifacts guaranteeing that calcium is globally conserved.

\section{Review of the different RyR behavior scenarios}

The ryanodine receptor is organized in clusters, (together with the LCC) the core of the Calcuim Release Unit. The opening of RyRs allows the calcium flow from the SR to the dyadic space. Besides the open configuration, the Ryanodine Receptor is known to have at least a closed state ready to open and an inactivated state. The different behavior of the RyR can be understood in terms of at least four different states as seen in Fig 1. The transition rates fix the general behavior of the receptor. It is generally agreed that the opening rate $K_{a}$ depends nonlinearly on the concentration of dyadic calcium itself. Calcium induces the release of calcium (CICR), normally considering a quadratic cooperativity $K_{a}=k_{o}\left(c_{d} / K_{c d}\right)^{2}$ where $c_{d}$ is the concentration of calcium in the dyadic space in the proximity of the
RyR cluster and $K_{c d} \simeq 100 \mu \mathrm{M}$.

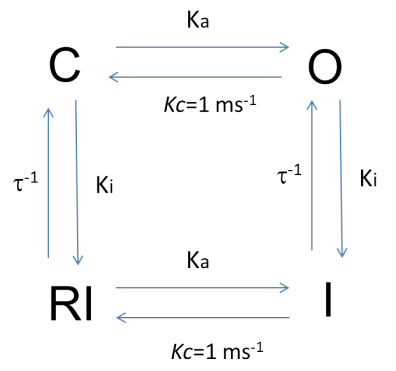

Figure 1. The 4-state RyR model . Changing the expression and the parameters of the transition rates from close (C) to open $(\mathrm{O}) K_{a}$, and from activated $(\mathrm{C}+\mathrm{O})$ towards inactivated $(\mathrm{RI}+\mathrm{I}) K_{i}, \tau$, one sets the different behavior scenarios for the Ryanodine Receptor, specially changing $\tau$ which fixes the recovery time from inactivation.

Besides that, different scenarios of how the RyR works have been proposed. Under a classic scenario the inactivation and recovery from inactivation rates are on the scale of $\mathrm{ms}$, leading to a rapid equilibium between the inactivated and activated states. We define this as the first scenario. It corresponds to the parameters indicated as set $\mathrm{I}$ in the table. This case was studied in [11] and has been shown to generate calcium alternans based on a highly nonlinear dependence of release on SR load. This appears due to the dependence of the release flux from the SR on SR load. A larger flux increases the dyadic calcium concentration that, in turn, opens the RyR, boosting even further SR Ca release.

In this paper, we address if other possible scenarios of the RyR lead to the same kind of transitions. Some authors have suggested (see [8] and references therein) that the RyR has a rather slow recovery from inactivation. Equilibration time between inactivated and closed states is comparable with the heart pacing rate. Inactivation in this scenario is normally considered to be dependent on calcium concentration $K_{i}=k_{i}\left(c_{d} / K_{c d}\right)$ with very low inactivation for pre-systolic calcium concentration of 0.1 $\mu \mathrm{M}$ but linearly increasing as dyadic calcium increases. This produces a self-inactivation of the RyR which is dependent on the strength of the calcium released. We define this as a second scenario using set II of parameters in the table. Finally, opening rates and inactivation rates may depend on calsequestrin concentration, making it directly dependent on SR load. Although this dependence is not needed to generate alternans with alternation in SR content, the following opening rate has been proposed $K_{a}=k_{o} G\left(c_{j S R}\right) c_{d}^{2}$ together with $K_{i}=k_{i} / G\left(c_{j S R}\right) c_{d}$ where the function $G$ is sigmoid [12]. In this paper, we use $G(x)=\frac{10+x^{2}}{1+x^{2}}$ with $x=c_{j S R} / 400(\mu \mathrm{M})$, as an example of this dependence (together with set III parameters). 
Table 1. Three different set of RyR2 transition rates used to test the universality of the calcium alternans transition.

\begin{tabular}{lccc}
\hline \hline RyR parameters & Set I & Set II & Set III \\
\hline Opening strength $k_{o} \mathrm{~ms}^{-1}$ & 2.1 & 2 & 0.2 \\
Inactivation strength $k_{i} \mathrm{~ms}^{-1}$ & 0.2 & 0.02 & 0.2 \\
Recovery time $\tau \mathrm{ms}$ & 1 & 400 & 400 \\
\hline \hline
\end{tabular}

\section{Results}

We find global alternans using the new set of parameters (II and III). Figure 2 shows global alternans in a fully stochastic model of calcium signaling where recovery from inactivation is slow. In Figure 2 the second set of parameters of the tables is used but similar results are obtained using the third set. Figure 2 shows alternation both in SR pre-systolic content and in the level of recovered RyRs. This reproduces the results obtained in analyzing this type of dynamics in whole-cell models. The graphs of Figure 2 also show how the ratio of inactivated versus activated states is not at equilibrium at the millisecond scale,i.e. it is not constant but presents a periodic dynamics linked to the pacing rate. More important, alternans are clearly global in nature and stochastic at the CaRU level, as clearly demonstrated in Figure 2 (right). On top, the average concentration of dyadic calcium presents clear and consistent alternans. A large transient alternates with a small transient. However, the three examples of the dyadic calcium concentration at the single $\mathrm{CaRU}$ level shows that there is no local persistent alternation. The release is locally stochastic, some beats present release and others do not. However, the coordination of a large amount of units makes that, at each beat, a majority of them release (strong beat) or not release it (weak beat).

This is more clearly seen in Figure 3 . We depict the different domains in a rather large cell of 200 by 200 units in a Z-plane. We compute at each unit the difference between the load of the junctional SR at the local level in one beat and subtract the value of this level at the previous beat. The strength of alternans is proportional to the difference in those to levels. However, this difference can be either positive or negative at a given beat. We code this difference going from red (strong sign positive alternans) to blue (strong sign negative alternans). The four different representations in Figure 3 correspond to simulations done at different pacing rates. We reproduce the same domain structure observed in [11]. The system presents areas with alternans in phase which do not lead to global alternans because different areas have different phases. The signature of these domains resembles the transitions observed in the Ising model. Although we have not cheked that the critical exponents are the same, the transition is clearly an
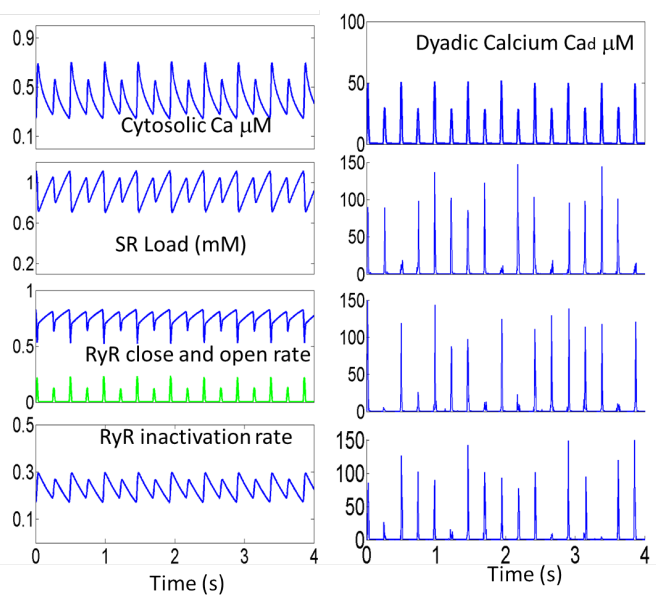

Figure 2. Using the first RyR set of parameters. On the left, from top to bottom, the average cytosolic and SR calcium concentrations showing alternans. Below, the ratio of RyR in the closed state $(\mathrm{C})$, open state $(\mathrm{O})$ and inactivated (RI plus I) . On the right, the average dyadic concentration on top. Below three examples of the dyadic concentration in three CaRU units in the cell. Global avarage arises from a coordination of partial domains of CaRUs. Each unit releases calcium stochastically.

order-disorder phase transition.

Another check of the nature of the order-disorder transition can be made testing if an order parameter presents the system size dependence expected in this type of transitions. We have performed simulation for system sizes 40 by 40 and 80 by 80 and computed the time average of the order parameter $m$, representing the global phase of oscillation. This parameter is defined locally at release unit $i$ and beat $n$ as $m_{i}(n)=\operatorname{sign}\left[(-1)^{n}\left(c_{j S R}^{i}(n)-c_{j S R}^{i}(n-\right.\right.$ $1))]$. Computing first the spatial average at beat $n$, taking its square value and then averaging over $n$ beats we obtain $<m^{2}>$ as a function of pacing rate. Figure 4 shows that it is clearly dependent of system size for the sets of parameters II and III. Notice however, how alternans using the third set of parameters is less spatially coordinated. It does not reach the level of full spatial ordering at pacing rates close to the bifurcation. We have found that introducing the SR dependence has a tendency to weaken the presence and strength of alternans. We have found parameters, for example strenghtening the $\mathrm{NaCa}$ ex-changer, where the third set of parameters does not present alternans while the first does. Contrary to intuition, direct SR dependence does not seem to help the presence of alternans due to SR nonlinearities.

In conclusion, we have found alternans as an orderdisorder phase-transition which coordinates local stochastic elements in three very different RyR behaviors. Consequently, we have found alternans where both the pre- 

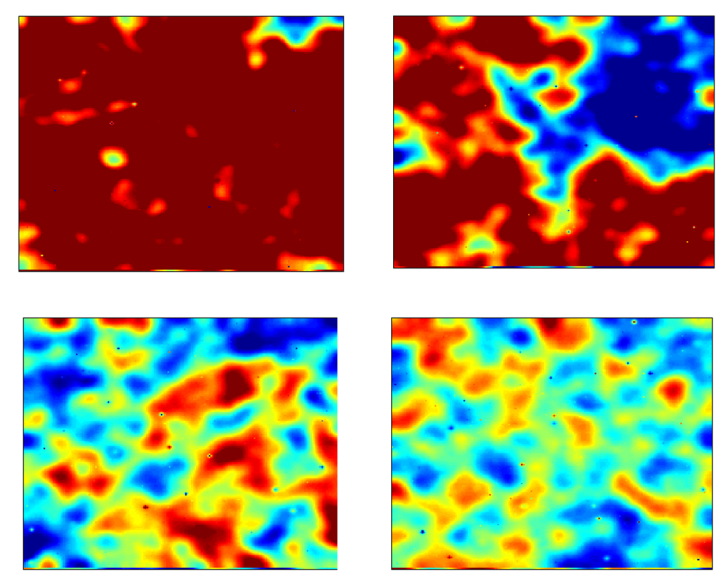

Figure 3. A map showing how different alternans domains form in a 200 by $200 \mathrm{CaRU}$ Z-plane at different pacing rates using the second set of parameters for the RyR. We measure the presystolic calcium load in one beat and subtract the same value of the previous beat. The color code is saturated in blue or red at absolute differences of 150 $\mu \mathrm{M}$. The pacing periods used in these graphs are, from left to right and up to bottom, 240, 260, 280 and $320 \mathrm{~ms}$. Domains are already present but are not coordinated and global alternans are not present.

systolic calcium load and the level of recovered RyR alternate. This demonstrates that the general picture developed in [11] holds. Alternans needs two key ingredients. First, a nonlinear local release probability and, second, coupling via diffusion. The particulars of the local non-linear behavior do not seem to affect the characteristics of the transition, as one would expect given the universal features of an order-disorder transition.

\section{Acknowledgements}

This work was supported by the Secretaria de Estado de Investigación, Desarrollo e Innovación (Spain), under grants FIS2011-28820-C02-01, SAF2014-58286-C2-2-R and the National Heart, Lung, and Blood Institute grant RO1HL101196.

\section{References}

[1] Amit G, Rosenbaum DS, Super DM, Costantini O. Microvolt T-wave alternans and electrophysiologic testing predict distinct arrhythmia substrates: implications for identifying patients at risk for sudden cardiac death. Heart Rhythm 2010 7(6):763-768.

[2] Weiss JN, Qu Z, Chen PS, Lin SF, Karagueuzian HS, Hayashi H, Garfinkel A, Karma A. The dynamics of cardiac fibrillation. Circulation 2005 112(8):1232-1240.

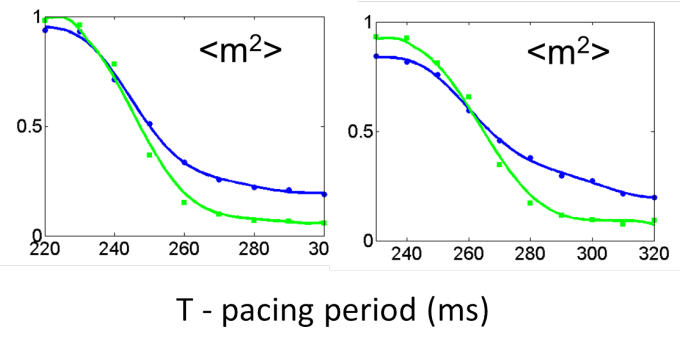

Figure 4. Order parameter of the transition as a function of the pacing period for two different set of RyR parameters. Set II was used on the left and set III on the right. We compute the order parameter for two different system sizes, 40 by 40 (blue) and a 80 by 80 (green) Z-planes after 20000 beats. The larger the system size, the steeper the transition becomes, a signature of system size dependence. The continuous line is a polynomial fit, guide to the eye

[3] Restrepo JG, Weiss JN, Karma A. Calsequestrin-mediated mechanism for cellular calcium transient alternans. Biophysical Journal 2008 95: 37673789.

[4] Clusin WT Calcium and cardiac arrhythmias: DADs, EADs, and alternans. Crit Rev Clin Lab Sci 2003 40(3):337-375.

[5] Weiss JN, Karma A, Shiferaw Y, Chen PS, Garfinkel A, Qu Z. From pulsus to pulseless: the saga of cardiac alternans. Circulation research 2006 98(10):1244-1253.

[6] Chudin E, Goldhaber J, Garfinkel A, Weiss J, Kogan B. Intracellular $\mathrm{Ca}(2+)$ dynamics and the stability of ventricular tachycardia. Biophysical Journal 1999 77(6):2930-2941.

[7] Restrepo JG, Karma. A Spatiotemporal intracellular calcium dynamics during cardiac alternans. Chaos 2009 19(3):037115.

[8] Alvarez-Lacalle E, Cantalapiedra IR, Peñaranda A, Cinca J, Hove-Madsen L, Echebarria B. Dependency of Calcium Al-ternans on Ryanodine Receptor Refractoriness. PLoS One 20138 (2), e55042

[9] Qu Z, Nivala M, Weiss JN Calcium alternans in cardiac myocytes: order from disorder. Journal of molecular and cellular cardiology 2013 58:100-109.

[10] Rovetti R, Cui X, Garfinkel A, Weiss JN, Qu Z Sparkinduced sparks as a mechanism of intracellular calcium alternans in cardiac myocytes. Circulation research 2010 106(10):1582-1591.

[11] Alvarez-Lacalle E, Echebarria B, Spalding J, Shiferaw Y. Calcium Alternans is Due to an Order-Disorder Phase Transition in Cardiac Cells. Physical Review Letters 2015 114 (10), 108101

[12] Shannon TR, Wang F, Puglisi J, Weber C, Bers DM. A mathematical treatment of integrated $\mathrm{Ca}$ dynamics within the ventricular myocyte. Biophysical Journal 2004 87(5):3351-71.

Address for correspondence:

enric.alvarez@upc.edu 